\title{
Genetic Encoding of a Highly Photostable, Long Lifetime Fluorescent Amino Acid for Imaging in Mammalian Cells
}

Chloe M. Jones, ${ }^{a, b}$ D. Miklos Robkis, ${ }^{a, b}$ Robert J. Blizzard, ${ }^{c}$ Mika Munari, ${ }^{d}$ Yarra Venkatesh, ${ }^{a}$ Tiberiu S. Mihaila, ${ }^{a}$ Alex J. Eddins, ${ }^{\mathrm{c}}$ Ryan A. Mehl, ${ }^{\mathrm{c}}$ William N. Zagotta, ${ }^{\mathrm{d}}$ Sharona E. Gordon, ${ }^{\mathrm{d}}$ and E. James Petersson ${ }^{\mathrm{b}, *}$

aDepartment of Chemistry, University of Pennsylvania, 231 South 34th Street, Philadelphia, PA 19104, USA.

bBiochemistry and Molecular Biophysics Graduate Group, University of Pennsylvania, 3700 Hamilton Walk, Philadelphia, PA 19104, USA.

'Department of Biochemistry and Biophysics, Oregon State University, 2011 Ag Life Sciences Building, Corvallis, Oregon 97331, USA.

dDepartment of Physiology and Biophysics, University of Washington, 1705 NE Pacific St., G-424, Box 357290, Seattle, WA 98195, USA.

* Email: ejpetersson@sas.upenn.edu

Acridonylalanine (Acd) is a fluorescent amino acid that is highly photostable, with a high quantum yield and long fluorescence lifetime in water. These properties make it superior to existing genetically encodable fluorescent amino acids for monitoring protein interactions and conformational changes through fluorescence polarization or lifetime experiments, including fluorescence lifetime imaging microscopy (FLIM). Here, we report the genetic incorporation of Acd using engineered pyrrolysine tRNA synthetase (RS) mutants that allow for efficient Acd incorporation in both $E$. coli and mammalian cells. We compare protein yields and amino acid specificity for these Acd RSs to identify an optimal construct. We also demonstrate the use of Acd in FLIM, where its long lifetime provides strong contrast compared to endogenous fluorophores and engineered fluorescent proteins, which have lifetimes less than 5 ns.

\section{Introduction}

Fluorescence spectroscopy provides a powerful method to monitor protein folding and dynamics in realtime under physiological conditions. One challenge for monitoring these dynamics is the ability to fluorescently label the protein of interest without disrupting its folding and function. ${ }^{1,2}$ Although widely used, large fluorescent proteins tags such as green fluorescent protein (GFP) ${ }^{3,4}$ can hinder native protein folding and function, 1,5,6 and affinity labeling such as bioorthogonal ("click") chemistry and SNAP ${ }^{8}$ or Halo ${ }^{9}$ tags can be limited by biocompatibility, labeling efficiency and solvent accessibility of the labeling location. ${ }^{1 \text {, }}$

${ }^{10}$ To avoid these issues, a fluorescent label can be incorporated co-translationally, eliminating the need for additional post-translational manipulation and allowing one to label proteins at interior sites. ${ }^{11}$ Incorporation of the labels in living cells is made possible by an engineered aminoacyl tRNA synthetase (RS) that selectively charges an orthogonal tRNA with a non-canonical amino acid (ncAA). ${ }^{12}$ The anticodon loop recognizes an amber stop codon that is introduced in the mRNA chain, leading to the insertion of the ncAA in the desired position of a protein of interest.

The Petersson and Mehl laboratories have previously incorporated acridonylalanine (Acd or $\delta$ ) in $E$. coli using these amber codon suppression methods. ${ }^{13-18} \mathrm{Acd}$ is a small fluorescent amino acid $\left(222 \AA^{3}\right)$, with an emission maximum at 420-450 nm, an extinction coefficient of 5,700 $\mathrm{M}^{-1} \mathrm{~cm}^{-1}$ and a quantum yield of 0.98 in aqueous buffer (ESI, Fig. S2 and Table S2) and high photostability (ESI, Fig. S1 and Table S1). ${ }^{19,20}$ We 
varies with the polarity of the environment and has been used in fluorescence polarization (FP) assays to investigate the dynamics of protein-protein interactions. ${ }^{17}$ Compared to other visible wavelength fluorescent ncAAs used for in cellulo genetic incorporation, ${ }^{22-25}$ hydroxycoumarin ethylglycine (Hco), coumarinyl lysine (CoulLys), dansylalanine (DanAla), and acetylnaphthalenylaminoalanine (Anap), Acd has the highest quantum yield, longest fluorescence lifetime, and greatest photostability in water (ESI, Figs. S1S9 and Tables S1-S6). The longer lifetime of Acd makes the fluorophore more responsive to molecular weight changes in FP experiments. ${ }^{17,26}$ It also permits better resolution of multiple populations in lifetime experiments, including single photon counting experiments ${ }^{27}$ and in fluorescence lifetime imaging microscopy (FLIM, Fig. 1). ${ }^{28}$
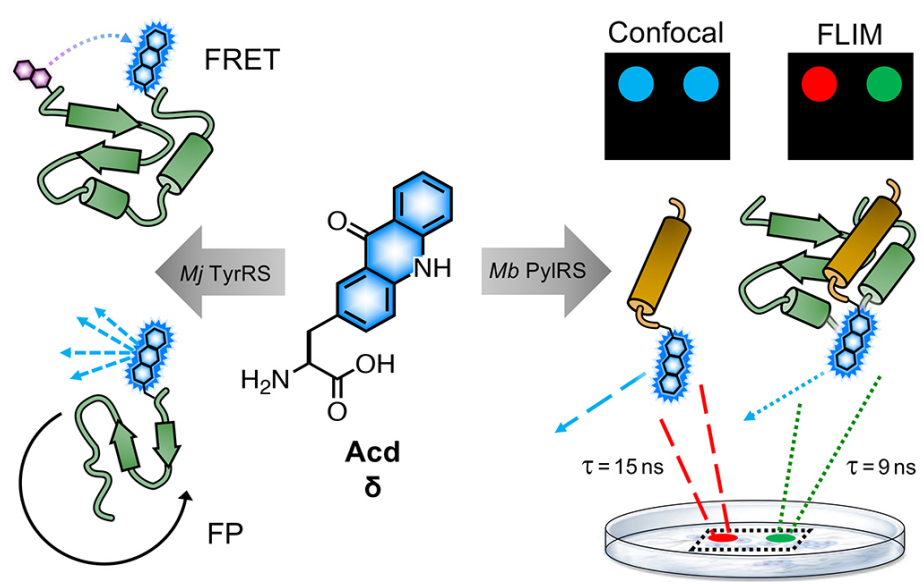

Fig. 1. Acd Applications. Acd (or $\delta$ ) has been previously used in Förster resonance energy transfer (FRET) and fluorescence polarization (FP) to monitor conformational changes and protein binding interactions among purified proteins expressed in $E$. coli using RSs based on $M j$ TyrRS. Development of a $M b$ PylRS-based system for mammalian cell incorporation enables confocal microscopy and fluorescence lifetime imaging (FLIM) studies to observe processes like protein association in live cells. FLIM pixels are coloured by lifetime, (red = $15 \mathrm{~ns}$, green $=9 \mathrm{~ns}$ ) which is related to the Acd local environment.

To date, all Acd studies relied on E. coli protein expression, as our engineered RSs were based on an Methanocaldococcus jannachii $(\mathrm{Mj})$ tyrosyl RS and thus were only orthogonal to the translation machinery in prokaryotes. In order to use Acd in mammalian cell imaging experiments, including FLIM, we needed an RS that is orthogonal in mammalian cells.

Here, we report the development of an Acd incorporation method that relies on an engineered pyrrolysine (Pyl) RS for site specific protein labeling. PyIRS variants from Methanosarcina bakeri $(M b){ }^{29}$ Methanosarcina mazei $(\mathrm{Mm}){ }^{29}$ and Methanomethylophilus alvus $(\mathrm{Ma})^{30,}{ }^{31}$ have been shown to be orthogonal in both $E$. coli and mammalian cells. Using engineered $M b$ PyIRS variants that specifically and efficiently incorporate Acd in both E. coli and mammalian cells, we also demonstrate that this new labelling platform can be used for FLIM studies in mammalian cells. 


\section{Results}

\section{Acd Synthetase Selection}

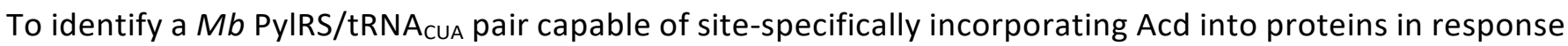
to an amber stop codon (TAG) we screened a library of $M b$ PyIRS variants in which five active-site residues were randomized to all 20 amino acids $\left(N_{311}, C_{313}, V_{366}, W_{382}, G_{387}\right)$ using a standard life/death selection in E. coli. ${ }^{32,33}$ Following a round of positive selection in the presence of Acd and a round of negative selection against canonical amino acids, 96 colonies were assessed for their ability to suppress a TAG codon interrupted sfGFP gene (sfGFP-TAG 150 ) in the presence of Acd and suppressor tRNA $A_{c U A}$. The top 25 performing clones were sequenced and 13 unique RS clones were identified with highly similar active site sequences (Fig. 2 Top and ESI, Table S7). These 13 clones were evaluated for (1) their efficiency (full-length protein yield in presence of Acd), (2) fidelity (level of canonical amino acid misincorporation in absence of Acd) and (3) their efficiency in producing full length protein using $N$-phenyl-amino phenylalanine (Npf), an undesired side product of Acd synthesis that was recognized by first-generation AcdRSs. ${ }^{13,14}$ Three of these clones showed permissivity for Npf, and were not considered further.

The most active and selective variants $(32,41,82)$ had similar mutations in the active site $\left(\mathrm{N}_{311} \mathrm{~S}\right.$ and $\mathrm{V}_{366} \mathrm{~A}$, as well as $\mathrm{W}_{382} \mathrm{~T}$ or $\left.\mathrm{W}_{382} \mathrm{~V}\right)$. $M b$ AcdRS 32 and 82 also had an additional active site mutation of $\mathrm{C}_{313} \mathrm{~A}$ or $\mathrm{C}_{313} \mathrm{G}$, respectively, and $\mathrm{Mb}$ AcdRS 82 also contained a non-active site mutation, L155V. AcdRS 41 and AcdRS 82 were selected for further evaluation as 82 was most active and 41 featured the same active site mutations, except it retained $\mathrm{Cys}_{313}$ of the parent RS. The two RSs were cloned into both pDULE2 ${ }^{35}$ and pUltra ${ }^{36}$ plasmids, under respective Ipp and tacl promoters, each also containing tRNAcUA. We did not retain the L155V mutation since other active sequences (e.g. 75) showed activity without the mutation. If necessary, Npf incorporation concerns can be eliminated by using an alternative Acd synthetic route. ${ }^{13}$

\section{Structural Modeling}

In order to better understand the effects of the mutations in the selected mutants, we built a homology model of $M b$ AcdRS 82 based on a x-ray crystal structure of substrate-bound Mm PyIRS (PDB ID: 2ZIN)..$^{34}$ With the exception of one loop region, there is very high active site sequence homology between the $\mathrm{Mm}$ and $M b$ PyIRS (ESI, Fig. S12), and it has been demonstrated that the conservation of the active site allows mutations for ncAA-selective variants to be swapped between the two types of PyIRS. ${ }^{37-41}$ Thus, we expect the homology model to be quite accurate. Acd was docked into the Mb AcdRS 82 active site in Rosetta, followed by relaxation of the structure (Fig. 2 Bottom) A model of $M b$ AcdRS 41 was generated by a $\mathrm{G}_{313} \mathrm{C}$ mutation of $M b$ AcdRS 82, followed by relaxation (ESI, Fig. S11). 
One can rationalize the effects of the four mutations in AcdRS 82 as follows: Asn $_{311}$ to Ser creates space in the active site and provides a hydrogen-bond acceptor for the Acd sidechain N-H, $\operatorname{Tr} p_{382}$ to Thr removes a steric clash and provides a hydrogen-bond donor for the Acd ketone, Cys $\mathrm{s}_{313}$ to Gly removes a steric clash with the distal ring of Acd and allows it to hydrogen-bond with both $\mathrm{Ser}_{311}$ and $\mathrm{Thr}_{382}$ together, and $\mathrm{Val}_{366}$ to Ala removes a steric clash. The existence of the hydrogen bond bridge in the AcdRS 82 model nicely explains its superior activity relative to AcdRS 41.
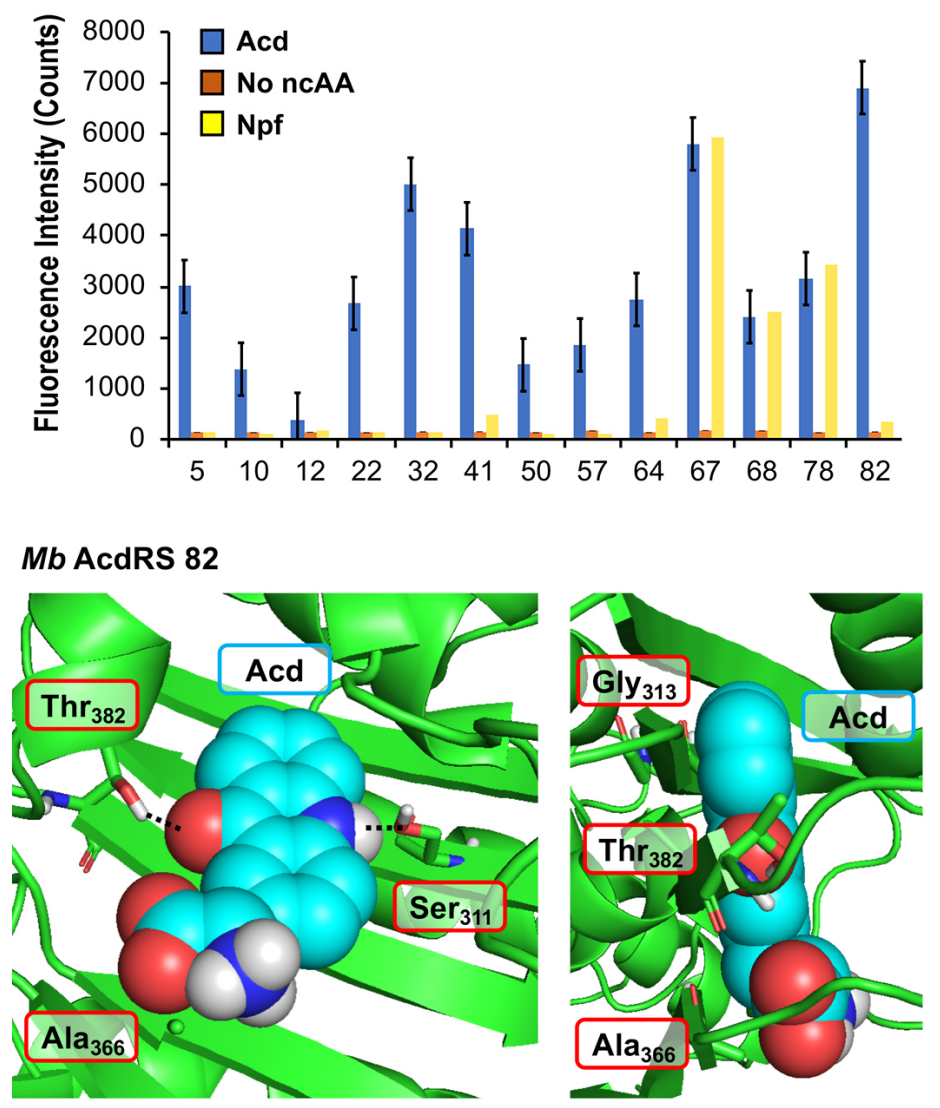

Fig. 2. PyIRS Mutants for Acd Incorporation. Top: Fluorescence measurements of sfGFP reporter to evaluate RS efficiency. Orange, blue, and yellow represent fluorescence from colonies induced in media containing no ncAA, $1 \mathrm{mM} \mathrm{Acd,} \mathrm{or} 1 \mathrm{mM}$ $\mathrm{Npf}$, respectively. Cultures of $500 \mu \mathrm{L}$ were grown for 48 hours before 4 -fold dilution of suspended cells with buffer for fluorescence measurement at $528 \mathrm{~nm}$ (485 nm excitation) using a platereader. RSs enabling high sfGFP fluorescence in Acd conditions with low fluorescence in no ncAA or Npf conditions were selected for further characterization. Additional data showing sfGFP fluorescence for all clones are shown in ESI, Fig. S10. Bottom: A homology model of $\mathrm{Mb}$ AcdRS 82 based on the x-ray crystal structure of $M m$ PyIRS (PDB ID: 2ZIN) ${ }^{35}$ with Acd docked in the active site using Rosetta. Key points of interaction with Acd are shown from two angles. Sites of mutation relative to parent $M b$ PyIRS are labeled in red boxes. A model of $M b$ AcdRS 41 is shown in ESI, Fig. S11. 


\section{Protein expression in E. coli and characterization of Acd incorporation}

From the initial screen, $M b$ AcdRS 41 and $M b$ AcdRS 82 were subjected to further investigation of their incorporation efficiency and ability to mis-incorporate canonical amino acids at the TAG site. To this end we expressed TAG-bearing constructs of $\alpha$-synuclein $(\alpha S)$ and calmodulin (CaM) to investigate the RSs in the context of an intrinsically disordered protein $(\alpha S)$ where the tertiary structure of the protein should not affect incorporation, and a structured protein (CaM) where destabilization by Acd incorporation could decrease protein expression. ${ }^{18}$ We have previously studied conformational changes and protein/protein interactions in both proteins using Acd. ${ }^{13,16}$ Each protein construct was fused to a C-terminal GyrA intein containing a six histidine $\left(\mathrm{His}_{6}\right)$ tag. This fusion construct provides a way to eliminate potential protein truncations, as the intein with the $\mathrm{His}_{6}$ tag is only produced for the full length protein and can be cleaved tracelessly using $\beta$-mercaptoethanol. ${ }^{42}$

To compare RS efficiency and specificity, we first expressed $\alpha \mathrm{S}$ using a plasmid encoding $\alpha \mathrm{S}$ with a TAG codon replacing Phe94 or Glu114 as well as a plasmid encoding either Mb AcdRS $41 M b$ AcdRS 82 and the

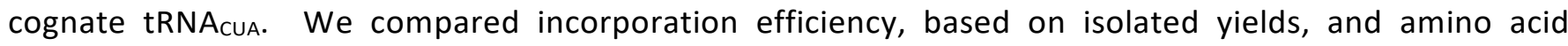
specificity, based on matrix-assisted laser desorption ionization (MALDI) mass spectrometry (MS) data. Phe94 and Glu114 were chosen as the incorporation sites as they have been shown to tolerate a variety of bulky fluorophore substitutions, including both ncAA incorporation and Cys modification. ${ }^{16,43-50}$ As a positive control, we expressed the same protein construct using an Mj RS previously optimized for Acd incorporation in E. coli (Mj AcdRS A9). ${ }^{14}$ As an additional comparison of anticipated masses and yields, we also expressed the wild-type (WT) $\alpha \mathrm{S}$ sequence fused to the His ${ }_{6}$ intein tag. This serves as an approximate reference mass for any mis-incorporation at the TAG site due to charging of canonical amino acids. Given the structural similarity of Acd to the aromatic amino acids Phe, Trp, and Tyr we anticipated that there was a potential for mis-incorporation of these amino acids. Indeed, Phe mis-incorporation has been previously observed in PyIRS mutants selected for incorporation of aromatic unnatural amino acids, ${ }^{52}$ although the selectivity of engineered PyIRSs has been reported to be generally good. ${ }^{53}$

Protein expressions in E. coli were performed with either pDULE2 or pUltra plasmids, using BL21 DE3 or C321.deltaA cells (release factor 1 removed and all 321 UAG codons changed to improve ncAA incorporation), ${ }^{54}$ respectively. Protein levels were monitored via gel throughout cell lysis, nickel purification and intein cleavage. SDS-PAGE analysis confirmed that full-length Acd-containing aS was produced for both $M b$ AcdRS 41 and Mb AcdRS 82 using either the pDULE2/BL21 and pUltra/C321 expression methods. However, MALDI MS analysis of purified whole protein constructs demonstrated that significant mis-incorporation of a canonical amino acid occurred with pUltra/C321 with either Mb AcdRS 41 and Mb AcdRS 82 (ESI, Fig. S19). In contrast, MALDI MS data confirmed that both Mb AcdRS 41 and Mb AcdRS 82 exclusively charge tRNA $A_{C U A}$ with Acd for pDULE2/BL21 expressions. (Fig. 3 and ESI, Figs. S17-S18). 
Whole protein masses matching the anticipated mass for Acd for incorporation at position 114 in $\alpha \mathrm{S}$ are shown in Table 1. No evidence of Npf incorporation was observed.
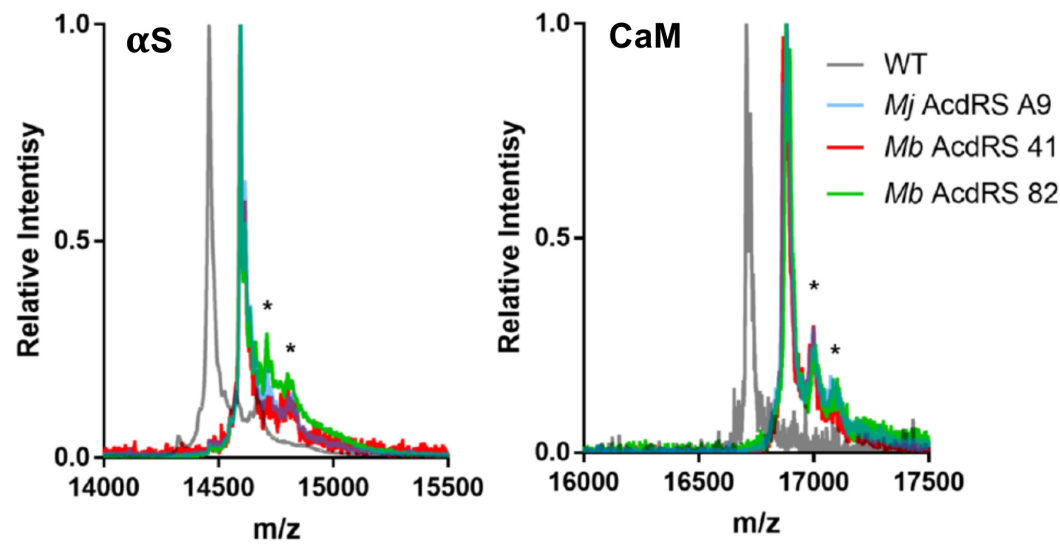

Fig. 3. MALDI MS Analysis of Acd Incorporation. Expressions of $\alpha S-E_{114} \delta$ or $C a M-L_{113} \delta$ using $M j$ AcdRS A9, $M b$ AcdRS 41 , or $M b$ AcdRS 82 were performed in parallel. WT $\alpha$ S and CaM were also expressed for comparison. MALDI MS data showed masses corresponding to Acd incorporation with no peaks corresponding to mis-incorporation of a canonical amino acid (approximately equal to WT mass). ${ }^{*}$ indicates a previously described MALDI matrix adduct. ${ }^{53}$

Table 1. Masses and yields of proteins from three rounds of $E$. coli Acd expression trials.

\begin{tabular}{|c|c|c|c|c|}
\hline Protein & Synthetase & 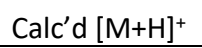 & Obsv'd $[\mathrm{M}+\mathrm{H}]^{+}$ & Relative Yielda \\
\hline WT $\alpha \mathrm{S}$ & $\mathrm{N} / \mathrm{A}$ & 14,460 & 14,459 & - \\
\hline$\alpha S-E_{114} \delta$ & Mj AcdRS A9 & 14,595 & 14,594 & $61 \pm 3 \%$ \\
\hline$\alpha S-E_{114} \delta$ & Mb AcdRS 41 & 14,595 & 14,594 & $6 \pm 1 \%$ \\
\hline$\alpha S-E_{114} \delta$ & Mb AcdRS 82 & 14,595 & 14,595 & $45 \pm 4 \%$ \\
\hline WT CaM & $\mathrm{N} / \mathrm{A}$ & 16,706 & 16,707 & - \\
\hline $\mathrm{CaM} \mathrm{L}_{113} \delta$ & Mj AcdRS A9 & 16,881 & 16,881 & $74 \pm 4 \%$ \\
\hline CaM- $\mathrm{L}_{113} \delta$ & Mb AcdRS 41 & 16,881 & 16,882 & $7 \pm 2 \%$ \\
\hline CaM- $\mathrm{L}_{113} \delta$ & Mb AcdRS 82 & 16,881 & 16,881 & $32 \pm 5 \%$ \\
\hline
\end{tabular}

a Yields based on Acd absorbance normalized to WT $\alpha \mathrm{S}$ or CaM expression, determined by Tyr absorbance, and confirmed by DC assay, as described in ESI.

Final protein yields were calculated based on molar absorptivity and confirmed by detergent compatible (DC) protein quantification assay, showing that Mb AcdRS 82 incorporates Acd into $\alpha S$ more efficiently than Mb AcdRS 41 (Table 1 and ESI, Tables S8-S9, gel analysis in Fig. S13-S16). The misincorporation of a natural amino acid under pUltra/C321 expression conditions may be a function of differences in culture media or the increased permissivity of the translation system in the C321 cells. ${ }^{54}$ No evidence of mis-incorporation was observed in multiple expression trials with the pDULE2/BL21 method. $\mathrm{CaM}-\mathrm{L}_{113} \delta$ yields in pDULE2/BL21 expression studies followed a similar trend for $M b$ AcdRS 41 and $M b$ AcdRS 82. Similar to $\alpha$ S expressed using pDULE2/BL21, no evidence of mis- incorporation was seen for either RS (Fig. 3). Protein yields calculated by molar absorptivity and confirmed by DC assay again showed 
that Mb AcdRS 82 is more active (Table 1 and ESI, Table S2). Together, the $\alpha \mathrm{S}$ and CaM results show that both $M b$ AcdRS 41 and Mb AcdRS 82 can incorporate Acd into proteins with high amino acid specificity using the pDULE2/BL21 expression system. However, Mb AcdRS 82 does this more efficiently to produce roughly 5-fold more purified protein. Excited by these high yields, we moved forward to study Acd incorporation in mammalian cells using Mb AcdRS 82. In addition, we wished to test whether Mb AcdRS 82 is more active than Mb AcdRS 41 in mammalian cells as it is in E. coli cells.

\section{Mammalian cell expression screening}

The incorporation efficiency of both of the $M b$ AcdRS variants was examined in mammalian cells. $M b$ AcdRS 41 and $M b$ AcdRS 82 were separately cloned into the PAcBac1 tR4-MbPyl vector, which contains two copies of a cognate $M b$ PyIRS tRNACUA. ${ }^{55}$ These plasmids were transfected into HEK293T/17 cells together with a dominant negative eukaryotic release factor one construct (DN-eRF1) that enhances ncAA expression yields in mammalian cells ${ }^{56}$ as well as a vector encoding enhanced GFP (EGFP) with a TAG codon at position $40\left(E G F P-Y_{40} \delta\right)$. RS efficiency and specificity were tested by measuring GFP fluorescence in the presence or absence of Acd for each Mb AcdRS. Transfections in the absence pAcBac1 tR4-AcdRS provided an additional negative control for read-through of the TAG codon in the absence of a cognate RS and tRNAcUA.

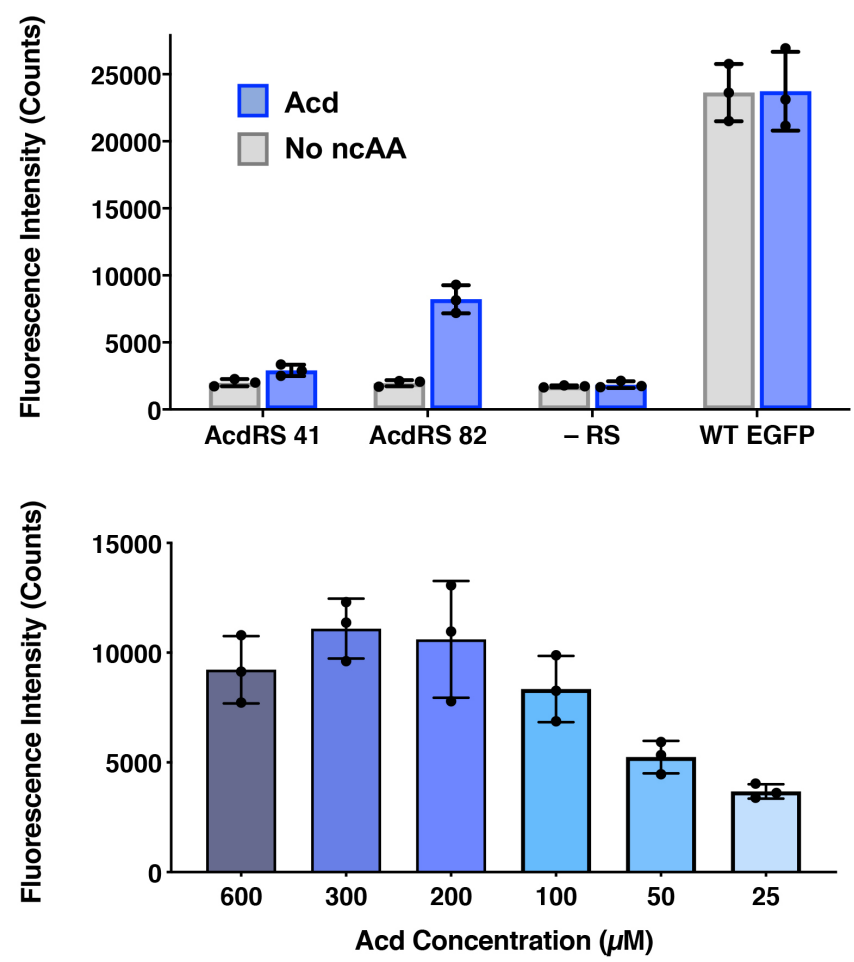

Fig. 4. Lysate Fluorescence Analysis of Acd Incorporation in Mammalian Cells. Top: Fluorescence measured from expression of EGFP- $_{40} \delta$ using $M b$ AcdRS 41 or 82 in HEK cells with $300 \mu \mathrm{M}$ Acd or no ncAA added. As a negative control, the EGFP plasmid was transfected without the AcdRS and tRNA CUA $_{\text {p }}$ plasmid (-aaRS). WT EGFP was separately transfected as a positive control. In all conditions, DN-eRF1 was also transfected. $n=3$. Bottom: Expression of EGFP-Y ${ }_{40} \delta$ using Mb AcdRS 82 was performed as above, with varying concentrations of Acd. $n=3$. Cell images are shown in ESI, Figs. S21-S22. 
The trend of incorporation efficiency for the RSs observed in E. coli held in that GFP fluorescence for Mb AcdRS 82 was 3-fold higher than Mb AcdRS 41. Additionally, both RSs showed low GFP fluorescence in the absence of Acd, indicating little to no mis- incorporation of natural amino acids (Fig. 4). Based on these results and our studies in $E$. coli, we selected Mb AcdRS 82 for further mammalian cell experiments. We also purified EGFP using a C-terminal $\mathrm{His}_{6}$ tag and analysed incorporation fidelity using electrospray ionization (ESI) MS. We found no evidence of misincorporation of Npf with either RS, but trace incorporation of a natural amino acid using AcdRS41 (ESI, Fig. S26). Based on these results and our studies in E. coli, we selected Mb AcdRS 82 for further mammalian cell experiments.

To determine optimal Acd concentrations for imaging experiments, we expressed EGFP- $Y_{40} \delta$ using AcdRS82 with varying concentrations of Acd. We found that maximal EGFP fluorescence was observed with $300 \mu \mathrm{M}$ Acd (Fig. 4, Bottom and images in ESI, Fig. S22). We also performed a cell viability study with varying Acd concentrations and found that Acd was toxic to cells at concentrations above $600 \mu \mathrm{M}$, possibly explaining the decrease in EGFP expression at $600 \mu \mathrm{M}$ Acd (ESI, Fig. S20). Cell imaging revealed that the cell morphology was normal at $300 \mu \mathrm{M}$ (ESI, Fig. S25). Finally, we varied the concentrations of transfection reagents and plasmids and tested the effect of DN-eRF1 to find optimal expression conditions (ESI, Figs. S23-S24). Comparing to WT EFGP expression based on both imaging and lysate fluorescence measurements in these experiments demonstrated that suppression was $29 \%$ efficient under optimal conditions, using Mb AcdRS 82 with DN-eRF1, $300 \mu \mathrm{M}$ Acd, and $1.6 \mu \mathrm{g}$ DNA (for a 6 well dish) at a 1:5 DNA to Lipofectamine 2000 ratio, with imaging after 24-48 $\mathrm{h}$.

\section{Mammalian cell fluorescence microscopy}

To investigate whether Acd can be used as a fluorescence reporter for protein localization in mammalian cells, we incorporated Acd at TAG mutants in three fusion proteins of varying localization, insulin receptor fused to GFP (IR-K $676 \delta$-GFP), hyperpolarization-activated cyclic nucleotide-gated ion channel fused to yellow fluorescent protein (spHCN-W ${ }_{355} \delta$-YFP), and maltose binding protein (MBP-E $E_{322} \delta$ ). The GFP and YFP fusions are C-terminal, so production of the fluorescent protein ensures that suppression of the stop codon was successful. Similar to the GFP screening transfection, Mb AcdRS 82 and DN- eRF1 plasmids were cotransfected into HEK293T/17 cells along with a vector encoding the protein of interest with a TAG codon at a site previously shown to permit bulky amino acids. ${ }^{57-59}$ After washout of unincorporated Acd, we imaged Acd-containing insulin IR- $\mathrm{K}_{676} \delta-\mathrm{GFP}, \mathrm{spHCN}-\mathrm{W}_{355} \delta-\mathrm{YFP}$, and MBP-E $\mathrm{E}_{322} \delta$ in live cells with confocal microscopy.

The localization of these proteins is known to be membrane bound in the case of IR-GFP and spHCNYFP, and cytosolic for MBP. Confocal imaging for IR-K ${ }_{676} \delta$-GFP and spHCN-W $W_{355} \delta$-YFP revealed that Acd fluorescence was localized to the membrane and colocalized with YFP or GFP fluorescence, respectively (Fig. 5). This demonstrates Acd's ability to be used as a fluorescent probe for protein localization. We 
additionally imaged cells containing the protein construct and Acd in the absence of the pAcBac1 tR4MbAcdRS82 plasmid (Fig. 5, no RS conditions). Confocal imaging revealed little or no fluorescence in the GFP or YFP channels, which indicates that no substantial readthrough of the TAG codon occurred. We observed only minor fluorescence in the Acd channel. This indicates that our washout protocols are effective and the remaining fluorescence may be due to endogenous fluorophores such as NADH or tRNA loaded with Acd, which cannot be washed out of the cell (wider fields of view shown in ESI, Fig. S27). Note that the rounded morphology of the cells is not a result of Acd toxicity, but of the extensive washing to remove Acd, as demonstrated by applying the same protocol to cells in the absence of Acd (ESI, Fig. S27). From these studies, we conclude that Acd incorporation is specific to our protein of interest and requires $M b$ AcdRS 82, as expected.

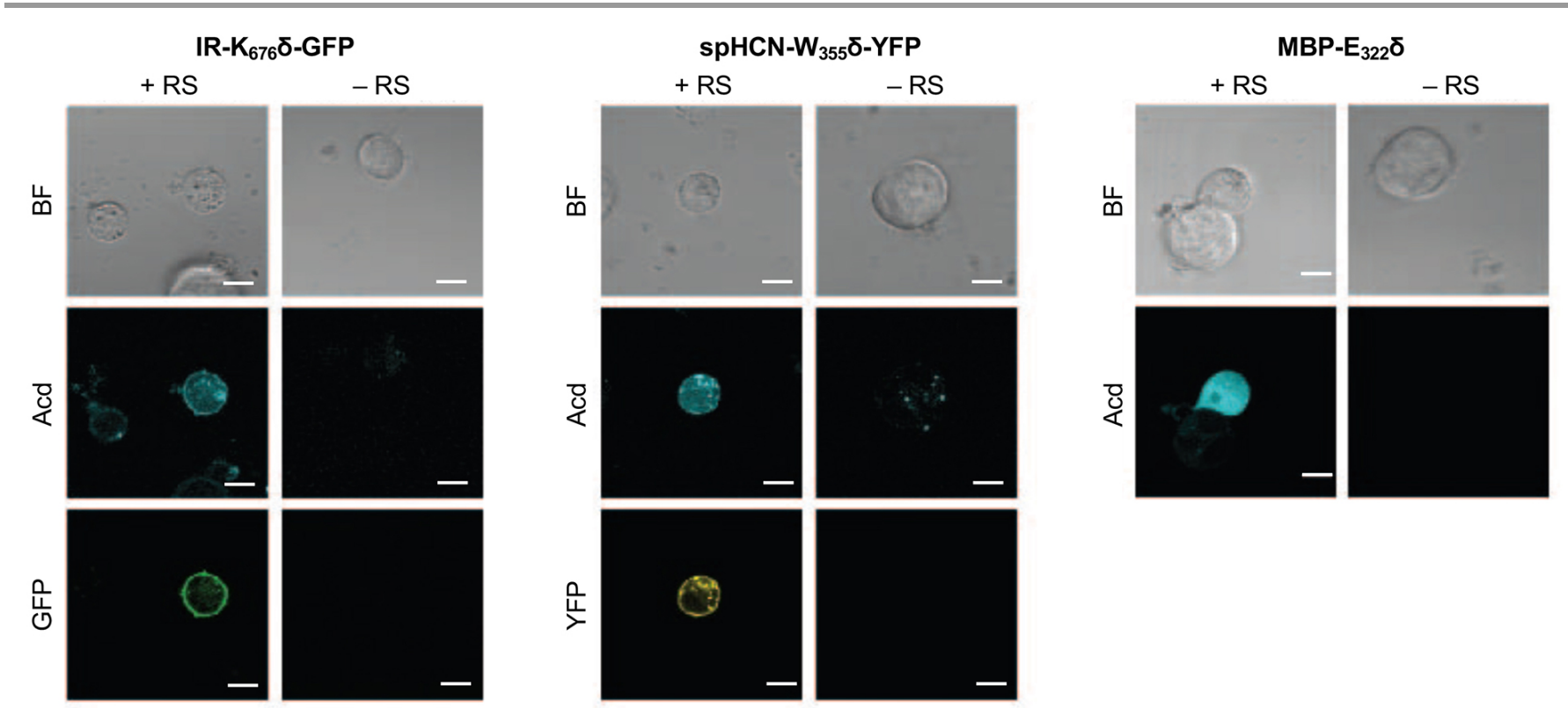

Fig. 5. Acd Incorporation into Proteins in Live HEK Cells. Cells were transfected with the indicated plasmid for the protein of interest with a TAG codon and a plasmid encoding a dominant negative release factor to suppress protein truncation, with (+ $\mathrm{RS}$ ) or without (-RS) a plasmid encoding $M b$ AcdRS 82 and tRNA $C \cup A$, Confocal microscopy images show brightfield (BF), the Acd fluorescence channel (405 $\mathrm{nm}$ excitation; 409-480 nm emission), and the GFP (488 $\mathrm{nm}$ excitation; 500-621 nm emission) or YFP (514 nm excitation; 519-621 nm emission) channels. Scale bar $=20 \mu \mathrm{m}$. 


\section{Mammalian cell fluorescence lifetime imaging}

Since Acd is particularly useful as a FLIM probe, as a proof of concept, we monitored the localization of Acd labeled insulin receptor (IR-K676 $\delta$-GFP) in HEK293T/17 cells using FLIM. FLIM spatially resolves the fluorescence decay rate of a sample to produce an image based on its fluorescence lifetime. Endogenous fluorophores typically found in a cell have a fluorescence lifetime in the range of $0.3-7.5 \mathrm{~ns}{ }^{28}$ Since Acd has a fluorescence lifetime of $15-16 \mathrm{~ns}$, our imaging method is free of background from endogenous cellular fluorophores. For this study, we transfected vectors containing $M b$ AcdRS 82, DN-eRF1, and IR- $\mathrm{K}_{676} \delta$-GFP into HEK293T/17 cells. Imaging the cells using intensity alone while exciting at $375 \mathrm{~nm}$ (Fig. 6) shows Acd fluorescence as well as autofluorescence, likely from NAD and NADH. Highlighting the pixels in which the majority of the intensity was due to Acd (lifetime $>15 \mathrm{~ns}$ ) in red allows the membrane localization of IR- $K_{676} \delta$-GFP to be discerned and also eliminates the autofluorescence signal. Highlighting the pixels with a short lifetime $(<4 \mathrm{~ns})$ in blue reveals the autofluorescence in all of the cells. The long lifetime pixels (red) compare well with emission from the GFP tag (excitation at $488 \mathrm{~nm}$ ). The pronounced autofluorescence signal in Fig. 6 compared to Fig. 5 is due to differences in the excitation wavelength used in each experiment. We note that the images in Fig. 6 seem to capture IR-K ${ }_{676} \delta$-GFP in different stages of maturation where it is observed in what appears to be ER and/or Golgi in addition to the plasma membrane. Additional FLIM data with spHCN-W $355 \delta$-YFP highlighting long lifetime Acd emission are shown in Fig. S28 in ESI.

IR-K 676 -GFP FLIM
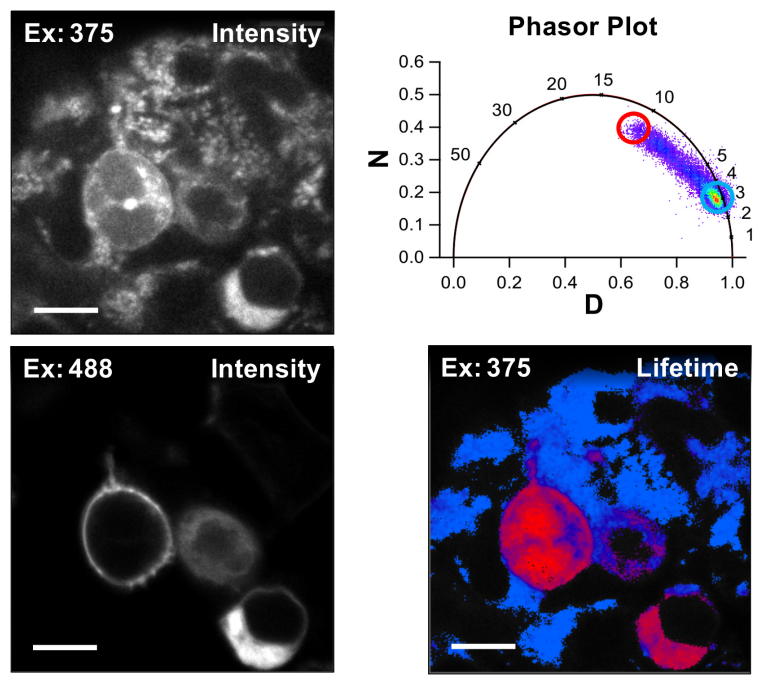

Fig. 6. Acd Fluorescence Lifetime Imaging (FLIM). FLIM allows Acd fluorescence to be separated from autofluorescence in living cells. IR-K $K_{676} \delta$-GFP was expressed with $M b$ AcdRS 82 in HEK cells. Intensity plots (left) show emission after excitation at 375 or $488 \mathrm{~nm}$. Phasor plot (top right) shows phase vectors D and N (see ESI for details) plotted for excitation at $375 \mathrm{nM}$ with $10 \mathrm{MHz}$ modulation and points representing lifetimes of pixels. Circles indicate pixels with a large fraction of lifetime $\geq 15 \mathrm{~ns}$ (red) and pixels with a large fraction of lifetimes $<4 \mathrm{~ns}$ (blue) in the lifetime image (bottom right). 


\section{Discussion}

The development of a PyIRS-based system for incorporating Acd is valuable for a number of reasons, including the applications in confocal microscopy and FLIM described here, as well as the potential for double-labelling of proteins using mutually orthogonal RSs deriving from PyIRS and Mj TyrRS. Acd has a higher quantum yield and greater photostability in buffer than any of the other genetically encodable ncAAs (ESI, Figs. S1-S9 and Tables S1-S6, quantum yield is comparable to Hco). Thus, Acd is in many ways superior for imaging, although this depends on the application. For example, the high level of environmental sensitivity depending on the protonation state of $\mathrm{HcO}$ is valuable for probing changes in local environment, but may confound other types of studies like FRET. Alternatively, the large Stokes shift of DanAla is useful for eliminating background scatter in spite of DanAla's very low quantum yield in water (0.10, see ESI, Table S2). Acd is particularly well-suited to FLIM studies because of its 15-16 ns lifetime in water, as most endogenous fluorophores have lifetimes $\leq 7$ ns allowing one to exclude their fluorescence by gating lifetime data. ${ }^{60}$ This makes Acd a superior FLIM probe compared to GFP variants, none of which have comparably long lifetimes $(<5 \mathrm{~ns}){ }^{61,62}$ The other genetically-encodable ncAAs all have shorter lifetimes, except DanAla in hydrophobic environments. However, the variation of the DanAla lifetime from 3 ns in water to 10-12 ns in MeOH, DMSO, and THF (ESI, Table S5) is a less useful range than that of Acd, which varies from $\sim 15 \mathrm{~ns}$ in water to $\sim 8 \mathrm{~ns}$ in organic solvent, only dropping to $\sim 4 \mathrm{~ns}$ in the very hydrophobic environment of THF (ESI., Table S3). Although we did not demonstrate it here, Acd's lifetime also makes it useful for anisotropy imaging, where the size range distinguishable by a fluorophore increases with fluorophore lifetime. ${ }^{63}$

Beyond FLIM applications, Mb AcdRS 82 can potentially be used to incorporate a photo-activatable version of Acd developed by Xiao and coworkers. ${ }^{64} \mathrm{Mb}$ AcdRS 82 could also be paired with an E. coli TyrRS engineered to encode $p$-azidophenylalanine in mammalian cells. ${ }^{65}$ This would allow in cellulo fluorescent labelling of a second site with "clickable dyes" opening access to site-specific controlled addition of FRET partners for Acd. Several strategies for encoding two amino acids have been published, including the use of ochre or opal codons, as well as four base codons. ${ }^{65-67}$ Chin and coworkers have also shown that one can generate mutually orthogonal RS/tRNA pairs from PyIRS variants. ${ }^{68}$ We will pursue these strategies where a coumarin derivative (Acd FRET donor) or BODIPY derivative (Acd FRET acceptor) could be attached. ${ }^{13}$ Finally, while Acd's properties are valuable, its low extinction coefficient and blue wavelength emission are not ideal for some microscopy applications, and we will attempt to obtain Mb AcdRS 82 variants that can encode brighter and/or red-shifted Acd analogs such as aminoacridonylalanine. ${ }^{21}$ We expect that the PyIRS may be better for this than the $M j$ TyrRS as it has been shown to accommodate many large amino acids. ${ }^{53}$ 


\section{Conclusion}

Acd has been previously demonstrated to be useful as a fluorescent marker and as a biophysical probe to monitor protein conformations without perturbing protein structure. Prior to this study, Acd labelling was limited to proteins expressed in E. coli and these proteins were used for in vitro biophysical experiments. Here, we have demonstrated the capability of two PyIRS variants to efficiently and selectively incorporate Acd into several proteins in E. coli and mammalian cells. Notably, the experiments were conducted in multiple laboratories and consistent results were observed. Mb AcdRS 82 afforded higher levels of protein expression in both cell types with excellent fidelity, as confirmed by MALDI and ESI MS, and was used for proof-of-concept fluorescence microscopy and FLIM studies. FLIM has advantages over confocal microscopy because it provides an additional dimension with which to discriminate changes in intensity due to the number of proteins from intensity changes due to solvatochromic effects resulting from conformational changes or interactions with other proteins. To our knowledge, the work described here is the first example of the direct use of a fluorescent ncAA in FLIM (the lone previous example used FRET with GFP). ${ }^{69}$ The long lifetime of Acd provides a large dynamic range for FLIM measurements, making it superior to GFP variants or the other genetically encodable ncAAs. This, in addition to Acd's high quantum yield and photostability, make Mb AcdRS 82 a valuable new tool for the protein imaging community, enabling facile labelling of proteins in mammalian cells with a small, non-perturbing fluorophore that has unique properties.

\section{Experimental}

\section{Aminoacyl tRNA synthetase (RS) screening}

Acd RS selection was performed on a library of $M b$ PyIRS mutants a life/death selection as previously described. ${ }^{70}$ Briefly, RS library members in a pBK screening plasmid were transformed into $E$. coli cells with a positive selection plasmid, pREP-pylT, containing an amber codon-disrupted chloramphenicol acetyltransferase gene and pyIRS tRNA (pylT). Cells containing the pBK-RS library and pREP-pylT were cultured in the presence of chloramphenicol and Acd. Surviving pBK RS library members were isolated and the pBK plasmid was transformed into cells containing a negative selection plasmid, pYOBB2-pylT, encoding an amber codon-disrupted barnase gene under control of an arabinose promoter as well as pylT. Cells containing PBK-RS library and pYOBB2-pyIT were cultured in the presence of arabinose, but in the absence of Acd. The remaining pBK-RS library from the surviving colonies was transformed into cells containing a GFP reporter plasmid, pALS-GFP-TAG ${ }_{150}$. Cells containing pBK-RS library and pALS-GFP-TAG 150 plasmids were grown in 96 well plates in autoinducing media (see ESI) containing $1 \mathrm{mM}$ Acd or Npf, or autoinducing media with no ncAA. GFP fluorescence was measured after $48 \mathrm{~h}$. Additional selection analysis and sequence information are provided in ESI.

\section{Computational modeling of Acd RS 82 active site}

M. mazei homologues of Acd RS 82 and 41 were identified by ClustalOmega alignment of MbPyIRS and MmPyIRS. A crystal structure of MmPyIRS bound to $N_{\varepsilon}$-Boc-L-Lysine and an adenosine triphosphate (ATP) analogue (PDB 2ZIN) was used as the basis for Rosetta modeling. ${ }^{34}$ Firstly, the ATP analogue was removed, and the amino acid ligand was replaced with Ala. Subsequently, missing loops were modeled using Rosetta Remodel and the structure was relaxed using the beta_nov16 scorefunction. ${ }^{71}$ Upon active site mutations, Acd was initially positioned using DARC and several relaxations of the structure were performed..$^{72}$ The final structure was analyzed in PyMol. ${ }^{73}$ 


\section{Protein expression and characterization of Acd incorporation}

Plasmids for each RS ( $M j$ AcdRS A9, Mb AcdRS 41, or Mb AcdRS 82) in the previously described pDULE2 vector, ${ }^{35}$ containing tRNA ${ }_{C \cup A}$, were separately transformed into BL21 cells that also contained the

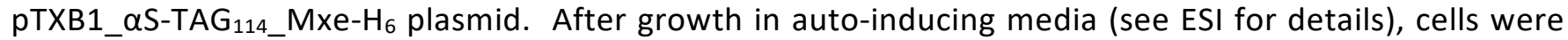
harvested by centrifugation. The supernatant was discarded and the pellet was resuspended, sonicated and pelleted, then the supernatant was collected and the protein purified with a $\mathrm{Ni}^{2+}$-NTA column. After elution, the protein was subjected to intein cleavage. Protein production and cleavage yields were analyzed by SDS PAGE. Each construct was further purified by fast protein liquid chromatography and analyzed by MALDI MS. The protein concentrations were analyzed by DC assay and UV-Vis absorbance. See ESI for CaM expression and purification details.

\section{Mammalian cell expression (EGFP screening)}

$M b$ AcdRS 41 and $M b$ AcdRS 82 were separately cloned into the pAcBac1 tR4-MbPyl vector to form pAcBac1 tR4-MbAcdRS41 and pAcBac1 tR4-MbAcdRS82. ${ }^{54}$ These plasmids were transfected into HEK293T/17 cells using Lipofectamine 2000, along with DN-eRF1 (peRF1-E55D.pcDNA5-FRT) and pEGFPY40TAG-C1 to evaluate suppression efficiency. Transfections were also performed in the absence of pAcBac1 tR4-MbAcdRS41 or pAcBac1 tR4-MbAcdRS82 as negative (-RS) controls. Transfections with DNeRF1 and pEGFP-C1 (WT EGFP) were used as positive controls. Transfections were performed with and without DN-eRF1 as well as with varying Acd concentrations, incubation times, and Lipofectamine 2000/DNA ratios to identify optimal expression conditions. Transfections were evaluated by imaging using an Olympus CKX53 microscope and by measuring the EGFP fluorescence of cell lysates using a Tecan M1000 plate reader.

\section{Mammalian cell expression (confocal microscopy)}

pAcBac1-tR4-MbAcdRS82 and DN-eRF1 plasmids were co-transfected into HEK293T/17 cells along with either pIR-TAG676-GFP, pcDNA3.1HE-spHCN-TAG355-YFP, or pcDNA3-FLAG-MBP1-E322TAG (FLAG-MBP1E322TAG-W340W.pcDNA3-k) plasmids. Confocal imaging .was performed on a Zeiss LSM 710 microscope in and analysed using Fiji software. ${ }^{74}$

\section{Fluorescence lifetime imaging microscopy (FLIM)}

FLIM experiments were performed at room temperature in DMEM supplemented with $10 \%$ FBS, penicillin, and streptomycin using an ISS Q2 laser scanner and ISS A320 FastFLIM system mounted on a Nikon TE2000U microscope. Acd was excited using a $375 \mathrm{~nm}$ laser and GFP and YFP were excited using bandpass filters to select lines of a YSL SC-PRO 7 supercontinuum laser. FLIM data were acquired and analysed using ISS VistaVision software.

\section{Plasmid and Acd Availability}

Sequences of the pDULE2-MbAcdRS82, for expression in E. coli, and pAcBac1-tR4-MbAcdRS82, for expression in mammalian cells, are given in the ESI. The plasmids will be made available via Addgene. Acd is available upon request to Prof. Petersson and is commercially available from Watanabe Chemicals.

\section{Conflicts of interest}

There are no conflicts to declare.

\section{Acknowledgements}

This work was supported by funding from the National Science Foundation (NSF CHE-1150351 to E.J.P, MCB-1518265 to R.A.M.) and the National Institutes of Health (NIH R01-NS103873 to E.J.P.; R01GM131168 to R.A.M.; R01-GM125351 and RO1-EY017564 to S.E.G.; R01-EY010329 and R01-GM127325 to W.N.Z.). Instruments supported by the NSF and NIH include: MALDI MS (NSF MRI-0820996) ESI MS (NIH S10-RR025628), FLIM microscope upgrade (NIH R01-GM125351-S1), and confocal microscope (NIH S10-RR025429). C.M.J. thanks the NIH for funding through the Structural Biology and Molecular Biophysics Training Program (T32 GM-008275). S.E.G. and W.N.Z. thank Edward Lemke for pIR-tAG-GFP 
cDNA, Peter Schultz for pEGFP-Y40TAG cDNA, and Jason Chin for peRF1-E55D.pcDNA5-FRT cDNA as well as Nathaniel Peters for assistance with confocal imaging. C321. $\triangle$ A.exp was a gift from George Church (Addgene plasmid \# 49018). We thank Abhishek Chatterjee for providing the pUltra-MbPyl-AcKRS and pET22b-T5 sfGFP plasmids. We thank Jeffrey Morré at the Mass Spectrometry Center at Oregon State University (OSUMSC) for ESI MS assistance.

\section{Notes and references}

1 L. C. Speight, M. Samanta and E. J. Petersson, Aust. J. Chem., 2014, 67, 686.

2 C. M. Haney, R. F. Wissner and E. J. Petersson, Curr. Opin. Chem. Biol., 2015, 28, 123.

3 B. A. Griffin, S. R. Adams and R. Y. Tsien, Science, 1998, 281, 269.

4 M. Chalfie, Y. Tu, G. Euskirchen, W. W. Ward and D. C. Prasher, Science, 1994, 263, 802.

5 K. Afitska, A. Fucikova, V. V. Shvadchak and D. A. Yushchenko, Biophys. J., 2017, 113, 2182.

6 D. W. Piston and G.-J. Kremers, Trends Biochem. Sci., 2007, 32, 407.

7 K. Lang and J. W. Chin, Chem. Rev., 2014, 114, 4764.

8 A. Keppler, S. Gendreizig, T. Gronemeyer, H. Pick, H. Vogel and K. Johnsson, Nat. Biotechnol., 2002, 21, 86.

9 G. V. Los, L. P. Encell, M. G. Mcdougall, D. D. Hartzell, N. Karassina, C. Zimprich, M. G. Wood, R. Learish, R. F. Ohana, M. Urh, D. Simpson, J. Mendez, K. Zimmerman, P. Otto, G. Vidugiris, J. Zhu, A. Darzins, D. H. Klaubert, R. F. Bulleit and K. V. Wood, ACS Chem. Biol., 2008, 3, 373.

10 J. Liu and Z. Cui, Bioconj. Chem., 2020, 31, 1587.

11 Z. Cheng, E. Kuru, A. Sachdeva and M. Vendrell, Nat. Rev. Chem., 2020, 4, 275.

12 A. Dumas, L. Lercher, C. D. Spicer and B. G. Davis, Chem. Sci., 2015, 6, 50.

13 L. C. Speight, A. K. Muthusamy, J. M. Goldberg, J. B. Warner, R. F. Wissner, T. S. Willi, B. F. Woodman, R. A. Mehl and E. J. Petersson, J. Am. Chem. Soc., 2013, 135, 18806.

14 I. Sungwienwong, Z. M. Hostetler, R. J. Blizzard, J. J. Porter, C. M. Driggers, L. Z. Mbengi, J. A. Villegas, L. C. Speight, J. G. Saven, J. J. Perona, R. M. Kohli, R. A. Mehl and E. J. Petersson, Org. Biomol. Chem., 2017, 15, 3603.

15 M. Padmanarayana, N. Hams, L. C. Speight, E. J. Petersson, R. A. Mehl and C. P. Johnson, Biochemistry, $2014,53,5023$.

16 J. J. Ferrie, N. leda, C. M. Haney, C. R. Walters, I. Sungwienwong, J. Yoon and E. J. Petersson, Chem. Commun., $2017, \mathbf{5 3 , 1 1 0 7 2 .}$

17 Z. M. Hostetler, M. B. Cory, C. M. Jones, E. J. Petersson and R. M. Kohli, ACS Chem. Biol., 2020, 15, 1127.

18 Z. M. Hostetler, J. J. Ferrie, M. R. Bornstein, I. Sungwienwong, E. J. Petersson and R. M. Kohli, ACS Chem. Biol., $2018,13,2855$.

19 H. Hamada, N. Kameshima, A. Szymanska, K. Wegner, L. Lankiewicz, H. Shinohara, M. Taki and M. Sisido, Bioorg. Med. Chem., 2005, 13, 3379.

20 A. Szymanska, K. Wegner and L. Lankiewicz, Helv. Chim. Acta, 2003, 86, 3326.

21 I. Sungwienwong, J. J. Ferrie, J. V. Jun, C. Liu, T. M. Barrett, Z. M. Hostetler, N. leda, A. Hendricks, A. K. Muthusamy, R. M. Kohli, D. M. Chenoweth, G. A. Petersson and E. J. Petersson, J. Phys. Org. Chem., 2018, 31, e3813.

22 J. Wang, J. Xie and P. G. Schultz, J. Am. Chem. Soc., 2006, 128, 8738.

23 A. Chatterjee, J. Guo, H. S. Lee and P. G. Schultz, J. Am. Chem. Soc., 2013, 135, 12540.

24 J. Luo, R. Uprety, Y. Naro, C. Chou, D. P. Nguyen, J. W. Chin and A. Deiters, J. Am. Chem. Soc., 2014, 136, 15551.

25 D. Summerer, S. Chen, N. Wu, A. Deiters, J. W. Chin and P. G. Schultz, Proc. Natl. Acad. Sci. USA, 2006, 103, 9785.

26 A. J. Pope, U. M. Haupts and K. J. Moore, Drug Disc. Today, 1999, 4, 350.

27 Y. Huang, J. J. Ferrie, X. Chen, Y. Zhang, D. M. Szantai-Kis, D. M. Chenoweth and E. J. Petersson, Chem. Commun., $2016,52,7798$.

28 M. Y. Berezin and S. Achilefu, Chem. Rev., 2010, 110, 2641.

29 W. Wan, J. M. Tharp and W. R. Liu, Biochim. Biophys. Acta, 2014, 1844, 1059.

30 B. Meineke, J. Heimgärtner, L. Lafranchi and S. J. Elsässer, ACS Chem. Biol., 2018, 13, 3087.

31 V. Beránek, J. C. W. Willis and J. W. Chin, Biochemistry, 2019, 58, 387.

32 H. S. Jang, S. Jana, R. J. Blizzard, J. C. Meeuwsen and R. A. Mehl, J. Am. Chem. Soc., 2020, 142, 7245.

33 J. J. Porter, H. S. Jang, E. M. Van Fossen, D. P. Nguyen, T. S. Willi, R. B. Cooley and R. A. Mehl, ACS Chem. Biol., $2019,14,1328$.

34 T. Yanagisawa, R. Ishii, R. Fukunaga, T. Kobayashi, K. Sakamoto and S. Yokoyama, Chem. Biol., 2008, $15,1187$.

35 S. J. Miyake-Stoner, C. A. Refakis, J. T. Hammill, H. Lusic, J. L. Hazen, A. Deiters and R. A. Mehl, Biochemistry, $2010,49,1667$.

36 A. Chatterjee, S. B. Sun, J. L. Furman, H. Xiao and P. G. Schultz, Biochemistry, 2013, 52, 1828.

37 R. J. Ernst, T. P. Krogager, E. S. Maywood, R. Zanchi, V. Beránek, T. S. Elliott, N. P. Barry, M. H. Hastings and J. W. Chin, Nat. Chem. Biol., 2016, 12, 776.

38 L.-T. Guo, Y.-S. Wang, A. Nakamura, D. Eiler, J. M. Kavran, M. Wong, L. L. Kiessling, T. A. Steitz, P. O'donoghue and D. Söll, Proc. Natl. Acad. Sci. USA, 2014, 111, 16724.

39 R. Serfling and I. Coin, in Methods in Enzymology, ed. V. L. Pecoraro, Academic Press, 2016, vol. 580, pp. 89.

40 K. Lang, L. Davis, S. Wallace, M. Mahesh, D. J. Cox, M. L. Blackman, J. M. Fox and J. W. Chin, J. Am. Chem. Soc., $2012,134,10317$.

41 N. Aloush, T. Schvartz, A. I. König, S. Cohen, E. Brozgol, B. Tam, D. Nachmias, O. Ben-David, Y. Garini, N. Elia and E. Arbely, Sci. Rep., 2018, 8, 14527.

42 S. Batjargal, C. R. Walters and E. J. Petersson, J. Am. Chem. Soc., 2015, 137, 1734.

43 C. M. Haney, C. L. Cleveland, R. F. Wissner, L. Owei, J. Robustelli, M. J. Daniels, M. Canyurt, P. Rodriguez, H. Ischiropoulos, T. Baumgart and E. J. Petersson, Biochemistry, 2017, 56, 683.

44 J. J. Ferrie, C. M. Haney, J. Yoon, B. Pan, Y.-C. Lin, Z. Fakhraai, E. Rhoades, A. Nath and E. J. Petersson, Biophys. J., $2018,114,53$.

45 C. M. Haney, R. F. Wissner, J. B. Warner, Y. X. J. Wang, J. J. Ferrie, D. J. Covell, R. J. Karpowicz, V. M. Y. Lee and E. J. Petersson, Org. Biomol. Chem., 2016, 14, 1584.

46 B. Pan, E. Rhoades and E. J. Petersson, ACS Chem. Biol., 2020, 15, 640.

47 C. M. Haney and E. J. Petersson, Chem. Commun., 2018, 54, 833. 
bioRxiv preprint doi: https://doi.org/10.1101/2021.04.05.438526; this version posted June 25, 2021. The copyright holder for this preprint

(which was not certified by peer review) is the author/funder. All rights reserved. No reuse allowed without permission.

48 M. J. Daniels, J. B. Nourse, H. Kim, V. Sainati, M. Schiavina, M. G. Murrali, B. Pan, J. J. Ferrie, C. M. Haney, R. Moons, N. S. Gould, A. Natalello, R. Grandori, F. Sobott, E. J. Petersson, E. Rhoades, R. Pierattelli, I. Felli, V. N. Uversky, K. A. Caldwell, G. A. Caldwell, E. S. Krol and H. Ischiropoulos, Sci. Rep., 2019, 9, 2937.

49 J. V. Jun, C. M. Haney, R. J. Karpowicz, S. Giannakoulias, V. M. Y. Lee, E. J. Petersson and D. M. Chenoweth, J. Am. Chem. Soc., 2019, 141, 1893.

50 R. J. Karpowicz, C. M. Haney, T. S. Mihaila, R. M. Sandler, E. J. Petersson and V. M. Y. Lee, J. Biol. Chem., 2017, $292,13482$.

51 S. Batjargal, Y. J. Wang, J. M. Goldberg, R. F. Wissner and E. J. Petersson, J. Am. Chem. Soc., 2012, 134, 9172.

52 J. M. Tharp, Y.-S. Wang, Y.-J. Lee, Y. Yang and W. R. Liu, ACS Chem. Biol., 2014, 9, 884.

53 T. Yanagisawa, M. Kuratani, E. Seki, N. Hino, K. Sakamoto and S. Yokoyama, Cell Chem. Biol., 2019, $26,936$.

54 T. M. Wannier, A. M. Kunjapur, D. P. Rice, M. J. Mcdonald, M. M. Desai and G. M. Church, Proc. Natl. Acad. Sci. USA, 2018, 115, 3090.

55 A. Chatterjee, H. Xiao, M. Bollong, H.-W. Ai and P. G. Schultz, Proc. Natl. Acad. Sci. USA, 2013, 110, 11803.

56 W. H. Schmied, S. J. Elsässer, C. Uttamapinant and J. W. Chin, J. Am. Chem. Soc., 2014, 136, 15577.

57 I. Nikić, J. H. Kang, G. E. Girona, I. V. Aramburu and E. A. Lemke, Nat. Protocols, 2015, 10, 780.

58 G. Dai, T. K. Aman, F. Dimaio and W. N. Zagotta, Nat. Struct. Mol. Biol., 2019, 26, 686.

59 S. E. Gordon, M. Munari and W. N. Zagotta, eLife, 2018, 7, e37248.

60 D. Rupsa, M. H. Tiffany, T. S. Joe, A. G. Amani and C. S. Melissa, J. Biomed. Optics, 2020, 25, 1.

61 K. J. Martin, E. J. Mcghee, J. P. Schwarz, M. Drysdale, S. M. Brachmann, V. Stucke, O. J. Sansom and K. I. Anderson, PLOS ONE, 2018, 13, e0183585.

62 L. Canty, S. Hariharan, Q. Liu, S. A. Haney and D. W. Andrews, PLOS ONE, 2018, 13, e0208075.

63 J. R. Lakowicz, Principles of Fluorescence Spectroscopy, Springer US, 3 edn., 2006.

64 J. Tang, C. Yu, A. Loredo, Y. Chen and H. Xiao, ChemBioChem, 2021, 22, 501.

65 Y. Zheng, P. S. Addy, R. Mukherjee and A. Chatterjee, Chem. Sci., 2017, 8, 7211.

66 H. Neumann, K. Wang, L. Davis, M. Garcia-Alai and J. W. Chin, Nature, 2010, 464, 441.

67 W. Wan, Y. Huang, Z. Wang, W. K. Russell, P.-J. Pai, D. H. Russell and W. R. Liu, Angew. Chem. Int. Ed., $2010,49,3211$.

68 J. C. W. Willis and J. W. Chin, Nat. Chem., 2018, 10, 831.

69 S. Brand and Y.-W. Wu, in Noncanonical Amino Acids: Methods and Protocols, ed. E. A. Lemke, Springer New York, New York, NY, 2018, DOI: 10.1007/978-1-4939-7574-7_21, pp. 327.

70 E. Arbely, J. Torres-Kolbus, A. Deiters and J. W. Chin, J. Am. Chem. Soc., 2012, 134, 11912.

71 P.-S. Huang, Y.-E. A. Ban, F. Richter, I. Andre, R. Vernon, W. R. Schief and D. Baker, PLOS ONE, 2011, 6, e24109.

72 R. Gowthaman, S. Lyskov and J. Karanicolas, PLOS ONE, 2015, 10, e0131612.

73 Schrödinger, The PyMOL Molecular Graphics System, Version 1.3, 2015

74 J. Schindelin, I. Arganda-Carreras, E. Frise, V. Kaynig, M. Longair, T. Pietzsch, S. Preibisch, C. Rueden, S. Saalfeld, B. Schmid, J.-Y. Tinevez, D. J. White, V. Hartenstein, K. Eliceiri, P. Tomancak and A. Cardona, Nat. Methods, 2012, 9, 676. 